\title{
Severe PID with sub-acute intestinal obstruction during third trimester of pregnancy: a rare presentation
}

\author{
Pesona Grace Lucksom*, Annet Thatal, Patrika Rai, \\ Spruha Navinbhai Thakkar, Kapil Singh Niranjan
}

Department of Obstetrics and Gynecology, Sikkim Manipal Institute of Medical Sciences (SMU), Sikkim, India

Received: 02 December 2018

Accepted: 07 January 2019

\section{*Correspondence:}

Dr. Pesona Grace Lucksom,

E-mail: pesonadoc@gmail.com

Copyright: ( $)$ the author(s), publisher and licensee Medip Academy. This is an open-access article distributed under the terms of the Creative Commons Attribution Non-Commercial License, which permits unrestricted non-commercial use, distribution, and reproduction in any medium, provided the original work is properly cited.

\begin{abstract}
Pelvic inflammatory disease (PID) during late pregnancy is rare. Authors report a case of severe PID manifesting as subacute intestinal obstruction at early third trimester of pregnancy. A 26 years woman at 28 weeks of gestation was admitted in OBG department of Central Referral Hospital (CRH), Sikkim for evaluation of pain in left hypochondrium with tachycardia and mild rise in temperature. Her WBC was $26,900 / \mathrm{cmm}$ while urine and blood cultures were sterile. USG showed single live gestation of 27 weeks 3 days with a heterogeneous mass suggestive of degenerative fibroid. She had recurrent episodes of subacute intestinal obstructions. Her condition deteriorated, hence, elective caesarean with exploratory laparotomy was done. Single live baby was delivered after which abdominal exploration revealed purulent ascites with multiple pockets of pus. Omental cake along with the bowel was adhered to the fundus of uterus. There was no area of perforation in the gut. Bilateral adnexa were adherent to the pre-sacral region which was densely adhered to underlying great vessels. Post operatively antibiotic to cover the anaerobic bacteria were given to which she responded. Baby died after 4 days of birth due to sepsis. One week after discharge she was readmitted with left sided pleural effusion which responded to antibiotics. PID should be considered a cause of abdominal pain and rise in temperature in pregnancy, as early diagnosis can be managed conservatively with antibiotics thus decreasing maternal and perinatal morbidity and mortality.
\end{abstract}

Keywords: Antibiotics, Infection, Pregnancy, Surgery

\section{INTRODUCTION}

Pelvic inflammatory disease (PID) is a spectrum of infection and inflammation of the upper genital tract organs typically involving the uterus (endometrium), fallopian tubes, ovaries pelvic peritoneum and surrounding structures. ${ }^{1}$ Pelvic inflammatory disease is a major problem to the reproductive health of young women as it is responsible for pain and infertility. Pelvic inflammatory disease is less common in pregnancy and is diagnosed often in the first and second trimesters. Its presentation in the third trimester is very rare and inflammatory disease leading to inflamed bowel loops and peritoneal abscess during late pregnancy is even rarer.

Diagnosis becomes difficult during the third trimester due to the enlarged uterus and pregnancy related changes and complications. Pregnant women suspected to have pelvic inflammatory disease are at high risk for maternal morbidity and preterm delivery. ${ }^{2}$ Conservative approach using antibiotics are recommended if diagnosed early, however, management varies depending on the clinical situation. Authors report a case of severe PID manifesting as sub-acute intestinal obstruction at early third trimester of pregnancy. 


\section{CASE REPORT}

A 26 years old antenatal woman at 28 weeks of gestation with history of previous caesarean, attended the obstetric outpatient department of central referral hospital (CRH), Sikkim, on $7^{\text {th }}$ November 2017 with history of pain in abdomen especially at the left hypochondrium on the fundus of the uterus along with giddiness and vomiting. She was previously admitted and treated with injectable antibiotics in another hospital for urinary tract infection. There was no relief in symptoms so had come to CRH for further opinion. She looked unwell and had mild rise in the body temperature on palpation, however, she stated that her body temperature was always mildly raised and thought it was normal. Her fundal height was 28 weeks with regular fetal heart with deep tenderness over the fundus of the uterus with no palpable mass. On admission her vitals were normal, WBC $26,900 / \mathrm{cmm}$, urine and blood cultures were sterile. USG showed single live gestation of 27 weeks 3 days with a heterogeneous mass of $7 \times 5 \mathrm{~cm}$ (suggestive of degenerative fibroid). These investigations and her clinical findings misled us to think of red degeneration of fibroid. After two days of admission she had acute pain abdomen and abdominal distension with vomiting. Surgical consultation was done, however, when the general surgeons came to examine her the distension subsided due to vomiting and their examination was hindered by the enlarged uterus. MRI done on $10^{\text {th }}$ November 2017 showed inflammatory bowel loops so was started on antibiotics. Her condition deteriorated, and she started having recurrent sub-acute intestinal obstruction. Plan for the need of laparotomy was discussed in detail with the patient and her family members as this surgery may lead to fetal morbidity and mortality, to which they agreed. Elective LSCS with exploratory laparotomy was done on $15^{\text {th }}$ November 2017. After opening the abdomen foul smelling purulent ascitic fluid was drained. Low transverse caesarean was done, and single live baby delivered. After closing the uterus abdominal exploration was done which revealed multiple pockets of pus in abdominal cavity (Right and Left pelvic, right paracolic, sub-hepatic area). Omental cake (due to slough) along with the bowel was adhered to the fundus of uterus. General surgeon was called to examine the gut for any signs of perforation, however, no area of perforation was identified. The area where she complained of severe pain was the area on the fundus where the small bowel was densely adhered. Adhesiolysis was done without injury to the bowel. Bilateral tubes and ovaries were not visualized as it was adhered to the sacral promontory region where it was densely adhered to underlying great vessels. Through a small space created laterally at the pelvis, one finger was inserted to examine the Pouch of Douglas and it was found to be normal. It was jointly decided by the Gynae and general surgeon not to dissect the para sacral area as it may lead to further complications. Infracolic omentectomy was done and specimen sent for Tuberculosis, culture sensitivity and histopathology examination. Thorough abdominal wash was given, and abdomen closed keeping a $32 \mathrm{Fr}$ pelvic drain. Specimen for culture sensitivity was negative for aerobic bacteria, hence, antibiotics were changed to cover the anaerobic bacteria after which she improved and was discharged on twelfth postoperative day. Specimens were also negative for tuberculosis and malignancy. However, her baby could not survive the sepsis and died after 4 days of birth. One week after discharge she complained of chest pain. Chest X-ray done showed left sided pleural effusion. She was readmitted in medicine department and investigated for TB which was negative, and she responded to antibiotics. She was a case of severe PID which must have been present before the pregnancy and flared up as pregnancy progressed. The immunocompromised state of pregnancy might have led to increase in severity leading to intestinal obstruction and pleural effusion.

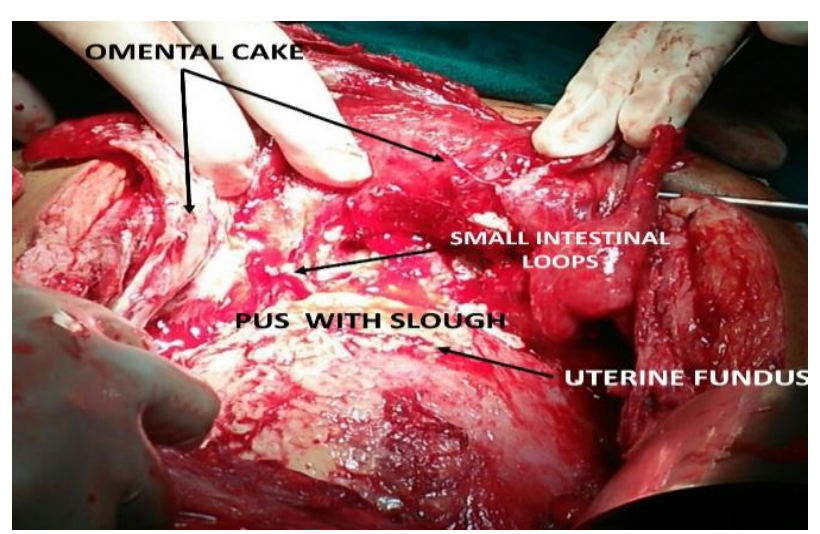

Figure 1: Intra-operative appearance of pregnant uterus with severe infection.

\section{DISCUSSION}

Pelvic inflammatory disease (PID) is a spectrum of infection and inflammation of the genital tract and is a disease of the reproductive age group. There is free anastomosis between the lymphatics and blood vessels in the pelvis which leads to the spread of infection from one pelvic organ to the other. Also, there is direct communication of the peritoneal cavity to the exterior through the vagina and the fallopian tube. Spread from non-reproductive organs, such as ruptured appendix and intestinal perforation, are also responsible for the pelvic infection. Pelvic inflammatory disease is usually responsible for infertility as it causes tubal block, dense adhesions or creates an unhealthy environment for the embryo to survive. Pelvic inflammatory disease is less common in pregnancy. Blanchard et al. found that acute salpingo-oophorities during pregnancy was more common in first trimester. ${ }^{3}$ Friedman and Bobrow proposed four mechanisms for pelvic inflammation during pregnancy. ${ }^{4}$ Firstly, hematogenous spread similar to pelvic tuberculosis, second route being lymphatic spread especially from vagina and cervix; thirdly, infection of a previously existing ovarian cyst and finally, flare-up of old infection. In some cases, cause of tuboovarian abscess may have an unknown origin. ${ }^{5}$ Pelvic 
inflammatory disease in the third trimester of pregnancy is rare and its presentation as sub-acute intestinal obstruction and peritoneal abscess complicating third trimester of pregnancy is rarer. There are very few case reports on pelvic abscess during third trimester of pregnancy. In this era of good obstetric care and good antibiotics such infections during third trimester are usually not seen. A case of recurrent pelvic abscess in pregnancy was reported by Sherer DM et al and in their review it was noted that the pelvic infection and pelvic abscess are less common in second and third trimester than in the first trimester. ${ }^{6}$ A case reported by Mahesha Navada resemble the present study, however, their patient did not present with such severe symptoms of recurrent sub-acute intestinal obstruction. ${ }^{7}$ Pelvic infection during pregnancy may occur either due to chronic pelvic infection existing before pregnancy or may be due to spread from the adjacent organs such as intestinal perforations as stated before, however, in our patient there were no signs of intestinal perforation or ruptured appendix. Pre-existing salpingitis or previously ruptured tubo-ovarian abscess could not be ruled out as there was dense adhesion of the bilateral adnexa over the presacral region. The woman's statement of her raised body temperature even before her pregnancy and the intraoperative finding of severe adhesions with multiple pockets of pus in the abdomen indicates the chronicity of the disease. The intraoperative findings along with pleural effusion is an indicator of severity of the disease. Pregnant women suspected to have Pelvic inflammatory disease are at high risk for morbidity and preterm delivery. ${ }^{2}$ Conservative management with antibiotics are preferred in pregnancy if infection is detected early but early detection is difficult as the symptoms of pelvic infections are masked by the pregnancy symptoms and the growing gravid uterus. Also, decision on the use of antibiotics in early pregnancy based on the early symptoms of pelvic inflammatory disease needs to be weighed against the risk involved to the fetus. Broadspectrum antibiotics given prior to delivery/surgery might mask neonatal infection or result in sterile blood culture though there is sepsis. It has also been studied that wrong choice of antibiotic may result in the baby being exposed to resistant strains of bacteria, which might lead to a worse neonatal outcome. ${ }^{8-10}$ In the recent studies there has been a shift in early neonatal sepsis from GBS to E. coli and other Gram negative organisms, and a change in resistance patterns, which may affect early gut colonization and has been implicated in early childhood asthma and allergy. ${ }^{8,10-15}$ Blood culture of the neonate in our case report was also sterile which may been due to the antibiotics used while trying conservative management and the baby died of severe sepsis.

Surgical drainage of ovarian abscess and conservative surgical approach under antibiotics is sometimes preferred depending on the clinical scenario. ${ }^{16}$ The medications and the effect of anaesthesia during surgery, however, increases perinatal morbidity and mortality. Pelvic inflammatory disease should be considered as a differential diagnosis of abdominal pain and rise in temperature even in pregnancy in spite of its rarity. If pelvic infection detected early in pregnancy, it should be managed with antibiotics so that such complications can be avoided, and we can improve maternal and fetal health. Surgical drainage under cover of antibiotics may sometimes be the only treatment options available depending on the severity.

\section{ACKNOWLEDGMENTS}

Authors would like to thank the Department of Surgery and $\mathrm{OBG}$ of $\mathrm{CRH}$ for their constant support and help while dealing with this case.

\section{Funding: No funding sources \\ Conflict of interest: None declared \\ Ethical approval: Not required}

\section{REFERENCES}

1. Wiesenfeld HC, Sweet RL, Ness RB, Krohn MA, Amortegui AJ, Hillier SL. Comparison of acute and subclinical pelvic inflammatory disease. Sex Transm Dis. 2005;32(7):400-5.

2. Pelvic Inflammatory Disease (PID)-2015 STD Treatment Guidelines Available at: https://www.cdc.gov/std/tg2015/pid.htm.

3. Blanchard AC, Weeks T. Pelvic inflammatory disease during pregnancy. South Med J. 1987;80(11):1363-5.

4. Friedman S, Bobrow LM. Pelvic inflammatory disease in pregnancy: a review of the literature and report of 5 cases. Obstet Gynecol. 1959;14(4):41725.

5. Laohaburanakit P, Treevijitsilp P, Tantawichian T, Bunyavejchevin S. Ruptured tuboovarian abscess in late pregnancy. A case report. J Reprod Med. 1999;44(6):551-5.

6. Sherer DM, Schwartz BM, Abulafia O. Management of pelvic abscess during pregnancy: a case and review of the literature. Obstet Gynecol Surv. 1999;54(10):655-2.

7. Navada HM, Bhat B. Pelvic inflammatory disease in the form of peritoneal abscess complicating late pregnancy. Case reports in Obstet Gynecol. 2011;2011.

8. American College of Obstetricians and Gynecologists. ACOG practice bulletin number 47, October 2003: Prophylactic Antibiotics in Labor and Delivery. Obstet Gynecol. 2003;102(4):875-2.

9. Edwards RK, Clark P, Sistrom CL, Duff P. Intrapartum antibiotic prophylaxis 1: relative effects of recommended antibiotics on gram-negative pathogens. Obstet Gynecol. 2002;100(3):534-9.

10. Terrone DA, Rinehart BK, Einstein MH, Britt LB, Martin JN, Perry KG. Neonatal sepsis and death caused by resistant Escherichia coli: possible consequences of extended maternal ampicillin 
administration. Am J obstet Gynecol. 1999;180(6):1345-8.

11. Bizzarro MJ, Dembry LM, Baltimore RS, Gallagher PG. Changing patterns in neonatal Escherichia coli sepsis and ampicillin resistance in the era of intrapartum antibiotic prophylaxis. Pediat. 2008;121(4):689-6.

12. Risk factors and opportunities for prevention of early-onset neonatal sepsis: a multicenter casecontrol study. - PubMed - NCBI. Available at: https://www.ncbi.nlm.nih.gov/pubmed/10617699.

13. Stoll BJ, Hansen N, Fanaroff AA, Wright LL, Carlo WA, Ehrenkranz RA, et al. Changes in pathogens causing early-onset sepsis in very-low-birth-weight infants. New Eng J Med. 2002;347(4):240-7.

14. Kozyrskyj AL, Ernst P, Becker AB. Increased risk of childhood asthma from antibiotic use in early life. Chest. 2007;131(6):1753-9.
15. Tan S, Holliman R, Russell AB. Hazards of widespread use of erythromycin for preterm prelabour rupture of membranes. Lancet. 2003;361(9355):437.

16. Yalcin OT, Tanir HM, Eskalen M. Unruptured pelvic abscesses in pregnancy: report of two cases. Gynecol Obstet Investigat. 2002;53(2):133-4.

Cite this article as: Lucksom PG, Thatal A, Rai P, Thakkar SN, Niranjan KS. Severe PID with subacute intestinal obstruction during third trimester of pregnancy: a rare presentation. Int J Reprod Contracept Obstet Gynecol 2019;8:754-7. 